\title{
Fast Multiple Access Selection through Variable Power Transmissions
}

\author{
Raymond Yim, Member, IEEE, Neelesh B. Mehta, Senior Member, IEEE, \\ and Andreas F. Molisch, Fellow, IEEE
}

\begin{abstract}
Many wireless applications demand a fast mechanism to detect the packet from a node with the highest priority ("best node") only, while packets from nodes with lower priority are irrelevant. In this paper, we introduce an extremely fast contention-based multiple access algorithm that selects the best node and requires only local information of the priorities of the nodes. The algorithm, which we call Variable Power Multiple Access Selection (VP-MAS), uses the local channel state information from the accessing nodes to the receiver, and maps the priorities onto the receive power. It is based on a key result that shows that mapping onto a set of discrete receive power levels is optimal, when the power levels are chosen to exploit packet capture that inherently occurs in a wireless physical layer. The VP-MAS algorithm adjusts the expected number of users that contend in each step and their respective transmission powers, depending on whether previous transmission attempts resulted in capture, idle channel, or collision. We also show how reliable information regarding the total received power at the receiver can be used to improve the algorithm by enhancing the feedback mechanism. The algorithm detects the packet from the best node in 1.5 to 2.1 slots, which is considerably lower than the 2.43 slot average achieved by the best algorithm known to date.
\end{abstract}

Index Terms-Multiuser diversity, splitting algorithms, multiple access, capture, collision, relay selection, power control.

\section{INTRODUCTION}

$\mathbf{M}$ ANY wireless multi-agent scenarios require the system to discover or select, from a set of nodes, the most suitable candidate for accessing an appropriate sink or base station. More formally, in these scenarios, we assign a realvalued "suitability metric" to each user, and aim to discover the user with the highest metric. For example, a system that exploits multiuser diversity allows the node with the "best" channel state (either absolutely in max-throughput scheduling, or relative to its average in proportional-fair scheduling [1]) to transmit so as to maximize the overall system throughput [2]. In a sensor network, a node can be selected to minimize total power consumption or maximize network lifetime. In cooperative communications, a transmitting source needs to select the best relay, among the many available relays, for collaboration [3], [4].

Manuscript received February 28, 2008; revised July 14, 2008 and October 17, 2008; accepted December 17, 2008. The associate editor coordinating the review of this paper and approving it for publication was V. K. N. Lau.

R. Yim is with Mitsubishi Electric Research Labs (MERL), Cambridge, MA, USA (e-mail: yim@merl.com).

N. B. Mehta is with the Indian Institute of Science (IISc), Bangalore, India.

A. F. Molisch is with the University of Southern California, Los Angeles,

USA. At the time of this work he was with MERL, Cambridge, MA, USA.

A part of this work was presented at ICC 2008.

Digital Object Identifier 10.1109/TWC.2009.080260
Due to the decentralized nature of wireless networks, the information necessary to evaluate the suitability metric is initially available only locally at the node itself and not anywhere in the network. Delivering all the information to a centralized node can be inefficient and consumes bandwidth resources that scale with the number of available nodes. Or, often, by itself, it constitutes a multiple access (MA) problem in which the best node wants to let the central node know its suitability metric. In either case, it is highly desirable that the process of selection be fast and decentralized. An attractive option for accomplishing this is a multiple access selection (MAS) mechanism in which the nodes themselves compete with each other based on their local information such that the best node is the first to successfully send its packet to the sink for further processing or identification. Once the node has been identified/selected by the sink, it can transmit data packets or be transmitted to (in multiuser diversity), or be used as the relay to forward data packets in cooperative communications. Depending on how the channel and the metric evolve over time, different nodes will get selected by MAS at different times; for example, improved fairness can be achieved by giving a higher priority (higher metric) to a node that has not transmitted for a long time. One key difference from conventional multiple access selection is that in the above scenarios, only the packet from the best node - and not other nodes - is of interest at any time.

Traditionally, studies of random multiple access communications rely on the assumption that a packet is successfully received by the information sink if and only if there is no concurrent transmissions. Based on this model, a plethora of multiple access schemes, such as ALOHA [5], carrier sensing multiple access [6], the first-come-first-serve (FCFS) algorithm [7], and the part-and-try algorithm [8], [9], have been proposed and their performance has been studied.

However, as has been well recognized in the literature, this collision model is a coarse and pessimistic way to model a wireless physical layer that handles interference. So long as the received power of a signal is sufficiently stronger than the interference power, the receiver can decode (capture) the stronger signal [10]. ${ }^{1}$ In fact, performance improvements due to such capture models have been shown in several systems such as ALOHA [13]-[15], 802.11 systems [16], Bluetooth radios [17], and cellular systems [18].

In this paper, we introduce and analyze an algorithm that

\footnotetext{
${ }^{1}$ This statement is valid even if no special measures for interference mitigation, such as multiuser detection [11] or smart antennas [12], are used.
} 
exploits the capture effect to speed up the detection of the best packet. As we shall see, capture with local channel knowledge significantly changes how MAS is done. In particular, the contributions of this paper are threefold: (i) First, we demonstrate that when the channel gain to the sink is known locally at the transmitting nodes, a fast, distributed algorithm can be found that controls how and which users transmit. We call this the Variable Power Multiple Access Selection (VPMAS) algorithm. (ii) We prove that monotonically mapping the metric into a set of discrete receive power levels is optimal. We derive an explicit characterization of the optimal mapping (called power-mapping function henceforth). (iii) We show that if the information sink has access to reliable information regarding the total receive power of each transmission, the performance can be enhanced even further using an algorithm called VP-MAS with Power-Splitting (VP-MAS-PS).

Since we assume that channel state information is available at each transmitter, our simulation results turn out to be independent of the underlying channel fading model. Instead, the results primarily depend on the dynamic range of the transmitting nodes. Since the goal of MAS is to only select the packet of the best user, the most important measure for the effectiveness of our algorithm is the average number of slots it takes to get the best-user packet to the information sink. Therefore, this metric was used for evaluating the multiple access splitting algorithm proposed in [19] and is also used in the current paper. This is unlike conventional multiple access, in which stability region [5], [7] analysis or packet delay analysis are of interest. We show that VP-MAS finds the best user in 2.1 and 1.8 slots on average when the receiver dynamic range is $20 \mathrm{~dB}$ and $40 \mathrm{~dB}$, respectively. For the same dynamic ranges, VP-MAS-PS finds the best user even faster in 2.0 and 1.5 slots, respectively. These results hold for an arbitrarily large number of contending users. In other words, the proposed MAS algorithm is always stable in that it is guaranteed to terminate and find the best user in finite time (when the metric has a continuous probability distribution).

\section{A. Related Work}

To the best of our knowledge, VP-MAS is the first algorithm for MAS that jointly exploits local channel state information and the capture effect. A number of previous papers have used components that are similar to some of the ones we exploit, though this is done in a different context. The Opportunistic Splitting Algorithm [19] achieves MAS by assuming the traditional contention model without capture, and finds the best node in 2.5 slots. Our result shows that the performance of MAS can be improved by at least $20 \%$ with additional information.

A back-off timer is another technique that is often used for MAS. In both [4] and [20], the suitability metric for cooperation, or the (perfect) local channel state information, is mapped into a timer that determines the start time of the signal transmission of each node. The better the metric, the shorter the backoff time. Reference [20] takes one step further in using the timing of beacon packets in determining the rates that enable MPR. Nonetheless, all these timing-based techniques suffer from the hidden-terminal and multipath problems inherent in wireless systems.
A promising generalization of capture called Multi-Packet Reception (MPR) has also been studied extensively in the literature [21]-[24]. These protocols differ in two important respects from our approach: (i) they aim to receive packets from all users, so their analyses do not apply to MAS; and (ii) they assume that the MAC protocol has no influence over the physical layer dynamics that specifies the multipacket reception probability matrix. As we shall see in this paper, one can indeed, in effect, gainfully influence the physical-layer probability of success.

Finally, the use of multiple receive power levels has been explored previously in [25] and [26]. While they only consider discrete power mappings, their conclusions are quite different from ours. Specifically, both papers show that if the receive power level and the data packet length are adjustable, then using a single receive power level achieves optimal throughput so long as the SINR threshold exceeds a certain value. On the other hand, we show in this paper that a larger receive power dynamic range coupled with gated contention that adapts to feedback always improves performance. The reasons for this seemingly contradictory conclusion are as follows: (i) The power levels in [25], [26] are selected at random and are not adjusted based on the outcome of the transmission. (ii) Their approach and analysis are based on a slottedALOHA type multiple access scheme per power level, whose goal of receiving data packets from all users is completely different from a MAS scheme like ours. A multi-power level slotted-ALOHA based approach was also proposed in [14], though again for receiving all packets, and their power levels were not adjusted based on feedback.

The remainder of the paper is organized as follows. In Sec. II, we describe the system model. An explicit characterization of the optimal metric-to-power mapping function is provided in Sec. III. Section IV describes the complete multipleaccess splitting algorithm. Simulation results in Sec. V are followed by our conclusions in Sec. VI.

\section{System Model}

We consider a wireless network consisting of $N \geq 2$ nodes and a message sink. We assume $N$ is known globally in the system. Each node, $i$, has a metric, $\mu_{i}$, that describes the priority of getting its packet to the sink. The sink is only interested in decoding the packet from the node with the highest priority; all other packets can be dropped. Furthermore, we assume that the priority metric remains constant during the multiple access selection process. $^{2}$ The metrics are independent real-valued random variables (RVs), with a probability distribution that is continuous and known globally in the system. The continuous probability distribution ensures that the probability that two metrics have exactly the same value is zero. ${ }^{3}$

We consider a time-slotted system. It is assumed that all contention packets have the same duration and transmission rate. Without loss of generality, the duration of a slot is set

\footnotetext{
${ }^{2}$ However, the metric can certainly take on different values for each new multiple access selection round.

${ }^{3}$ The case where the actual metric of the system is discrete in nature, for example, when it is determined by quality of service (QoS) parameters, can be handled by making each node generate a random number based on its metric. This creates an effective metric with a continuous probability distribution.
} 
to unity. The channel gain between node $i$ and the message sink is denoted by $h_{i}$, and is assumed to be known at the transmitter, as was assumed in channel-aware Aloha [27], [28].

Let $P_{i}$ denote the power received from node $i$. (We shall henceforth call it 'receive power'.) The sink can decode the packet from node $i$ successfully if the received signal to interference and noise ratio (SINR) exceeds a threshold:

$$
\frac{P_{i}}{\sum_{j \neq i} P_{j}+\sigma^{2}} \geq \bar{\gamma}
$$

where $\sigma^{2}$ is the noise power, and $\bar{\gamma} \geq 1$ is a threshold that depends on the modulation and coding used for packet transmission[29]. ${ }^{4}$ Thus, a packet can be decoded successfully even when two or more users transmit simultaneously, if their levels are sufficiently apart; otherwise, none of the packets can be captured.

\section{A. Controlling Receive Power}

The ability to control receive power is crucial to the development of a fast multiple access selection algorithm that takes advantage of capture in (1). To do so, the message sink can occasionally broadcast a (predefined) pilot sequence. ${ }^{5}$ Each node then locally computes its channel gain to the sink. For a target receive power $P$ and an estimated channel gain $h$, a node transmits its message at power $P / h$. This technique is analogous to the power control that is ubiquitous in secondand third-generation CDMA-based cellular systems [30], [31].

In this work, we assume that the power received from any node lies between $P_{\min }$ and $P_{\max }$, where $P_{\max }$ is obtained by considering the hardware limitations at the receiver. The dynamic range of the received power can be in tens of decibels, depending on the difference between the best and worst channel gains in the system as well as the dynamic range of the transmitters. For example, the mobile station transmit power dynamic range is about $34 \mathrm{~dB}$ in GSM systems [30] and $74 \mathrm{~dB}$ in WCDMA systems [31].

\section{B. Channel Fading}

Due to channel fading, it is possible that the maximum receive power of packets from some nodes are below $P_{\max }$ even when they are sent at maximum transmit power. In this case, these nodes are considered to be out of range, and, therefore, excluded from the discussion. Admission control mechanisms to ensure this are beyond the scope of this paper. Otherwise, we assume that the fading characteristic of the channel is slow enough so that the channel estimation allows the nodes to accurately control the receive power as described in the previous subsection, and the channel remains constant throughout the duration of multiple access selection. As we shall see, this assumption is easily satisfied since the selection process is very fast.

\footnotetext{
${ }^{4}$ In a CDMA system, the SNR considered here will correspond to the SNR after the despreading operation at the receiver. Thus, $\bar{\gamma} \geq 1$ is still the reasonable operating range.

${ }^{5}$ The frequency of estimation depends on the coherence time of the channel.
}

\section{Multiple Access Selection Process And Feedback}

At the beginning of a time slot, each node independently decides, depending on criteria that will be specified later, whether or not to transmit its packet. If it does transmit, it sets the receive power of its packet according to a metric-toreceive power mapping $\pi(\cdot)$. That is, it ensures that its signal is received with a power level $\pi(\mu)$, which depends on its metric. (Therefore, the node will transmit at a power of $\pi(\mu) / h_{i}{ }^{6}$ ) At the end of every slot, one of three outcomes is broadcast to all nodes. If no node transmits in the slot, the outcome is idle. If the received powers are such that the signal of one of the transmitting nodes can be captured (as per (1)), the outcome is a success. Otherwise, if none of the transmitted signals can be captured, the outcome is a collision. This can happen, for example, when the best two nodes transmit and do so at power levels that are not sufficiently apart to enable the best node's packet to be captured. The base station broadcasts this outcome at the end of every slot.

\section{FUndAMENTAL INSIGHTS}

Our aim is to design a fast multiple access selection algorithm such that the packet sent by the best node is the first to be successfully decoded by the message sink. Before describing the complete algorithm, we first consider the metric-to-receive power mapping for the case in which exactly two nodes simultaneously transmit. We then generalize it to the case in which a fixed, but arbitrary, number of nodes transmit. Finally, the complete VP-MAS algorithm is developed in the following section.

\section{A. Simultaneous Transmissions from Two Nodes}

Consider the scenario where exactly two nodes, $a$ and $b$, with corresponding metrics $\mu_{a}$ and $\mu_{b}$, transmit their packets simultaneously. We first assume that the metrics are uniformly distributed in the range $\left[\mu_{\min }, \mu_{\max }\right)$, and will generalize the result to arbitrary probability distributions later. Consider an order statistic of the metric $\mu$ such that $a$ indexes the node with the smaller of the two metrics (i.e., $\mu_{a}<\mu_{b}$ ).

To begin, we want to find the optimal function $\pi(\cdot)$ that maximizes the probability that the sink decodes the packet transmitted by $b$ successfully. This is an infinite dimensional optimization problem, which can be stated mathematically as:

$$
\max _{\pi} \operatorname{Pr}\left\{\frac{\pi\left(\mu_{b}\right)}{\pi\left(\mu_{a}\right)+\sigma^{2}} \geq \bar{\gamma}\right\}
$$

subject to

$$
P_{\min } \leq \pi(\mu) \leq P_{\max } .
$$

It is important that the function, $\pi($.$) , be set so that a user$ with a lower metric does not get selected when a user with the higher metric has also transmitted. If a function does not violate such a condition, we say that it is valid. The following lemma, which follows from (2), characterizes the space of all valid power mappings:

\footnotetext{
${ }^{6}$ The assumption in [25], [26] that the users are equidistant from the sink, while less general, also ensures that the transmit power can be set to achieve a desired receive power level.
} 
Lemma 1: A mapping $\pi(\cdot)$ is valid for MAS iff $\pi\left(\mu_{a}\right)<$ $\bar{\gamma}\left(\pi\left(\mu_{b}\right)+\sigma^{2}\right)$ for all $\mu_{a}<\mu_{b}$.

Since $\bar{\gamma} \geq 1$, this implies that all monotonic non-decreasing (MND) functions are valid power mappings. However, the lemma also indicates that there exist valid mappings that are not MND, i.e., $\pi\left(\mu_{a}\right)>\pi\left(\mu_{b}\right)$ for some $\mu_{a}<\mu_{b}$. One trivial example occurs when $P_{\max }<\bar{\gamma}\left(P_{\min }+\sigma^{2}\right)$. In this case, all functions are valid (and optimal) as they all result in a zero probability of success, which is the only value possible.

Since the goal of the multiple access algorithm is to maximize the success probability of decoding the packet transmitted by the node with the largest metric, it is intuitive to consider only MND functions. The following lemma formalizes this intuition and shows that an optimal solution can indeed be found in the space of MND functions.

Lemma 2: An optimal mapping $\pi(\cdot)$ can be found in the space of MND functions.

Proof: Let $\pi_{\text {opt }}(\mu)$ be an optimal mapping that is not necessarily MND. We can "sort" this function by considering its cumulative distribution function when $\mu$ is uniformly distributed in the range $\left[\mu_{\min }, \mu_{\max }\right)$. That is, consider the transformation

$$
\begin{aligned}
& \pi\left(\mu^{\prime}\right)=P_{\min }+\left(P_{\max }-P_{\min }\right) \times \\
& \operatorname{Pr}\left(\pi_{\text {opt }}(\mu)<P_{\min }+\left(P_{\max }-P_{\min }\right) \frac{\mu^{\prime}-\mu_{\min }}{\mu_{\max }-\mu_{\min }}\right),
\end{aligned}
$$

for $\mu^{\prime} \in\left[\mu_{\min }, \mu_{\max }\right)$. Then, $\pi\left(\mu^{\prime}\right)$ is MND and has the same power distribution as $\pi_{\text {opt }}(\mu)$. Such a sorting clearly does not affect the probability of success.

The following important lemma proves that an optimal MND, in fact, maps the metrics into a set of discrete power levels.

Lemma 3: Let $\pi(\cdot)$ be a function that maps the metrics into $(L+1)$ discrete power levels in the set $\mathbf{Q}=\left\{q_{0}, q_{1}, q_{2}, \cdots, q_{L}\right\}$ such that

$$
L=\left\lfloor\log _{\bar{\gamma}}\left(\frac{(\bar{\gamma}-1) P_{\max }+\sigma^{2} \bar{\gamma}}{(\bar{\gamma}-1) P_{\min }+\sigma^{2} \bar{\gamma}}\right)\right\rfloor,
$$

and

$$
q_{i}=\bar{\gamma}^{i} P_{\min }+\sigma^{2} \bar{\gamma} \frac{\bar{\gamma}^{i}-1}{\bar{\gamma}-1}, \quad 0 \leq i \leq L .
$$

Then $\pi(\cdot)$ optimizes the probability of success in (2).

Proof: The proof is given in Appendix A.

The power levels shown in (6) result from setting $q_{0}=P_{\min }$, and minimizing the gap between the adjacent power levels. The number of levels depends on the dynamic range $P_{\min }$ and $P_{\max }$.

It is important to note that the above lemma does not imply that an optimal function must be discrete. In other words, while the above discrete solution is optimal, it need not be the uniquely optimal solution. For example, when $q_{L}<P_{\max }$, the highest power level can be increased without affecting the probability of success. Furthermore, appropriately scaling the power levels while still ensuring that there are $(L+1)$ of them below $P_{\max }$ also ensures the same probability of success and results in a different optimal solution.

Let users with metrics in the range $\left[m_{i}, m_{i+1}\right)$ be mapped to the receive power $q_{i}$, for $0 \leq i \leq L$, with $m_{0}=\mu_{\min }$ and

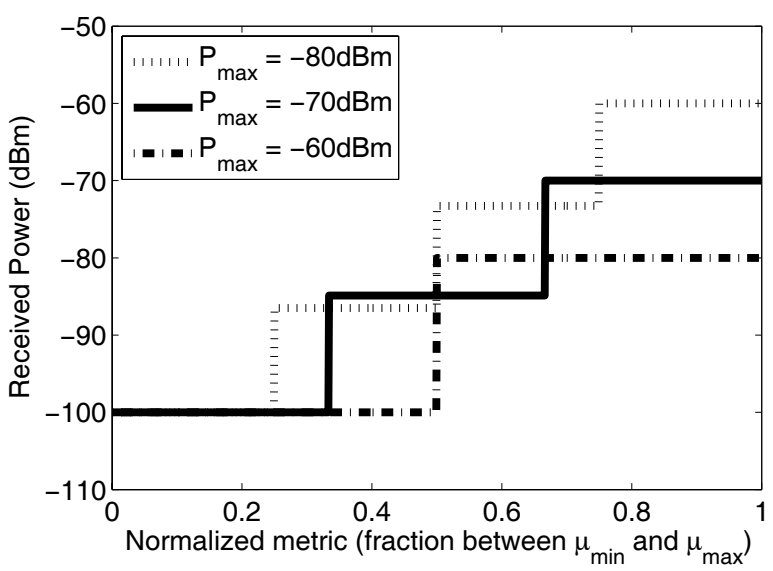

Fig. 1. Receive powers as a function of the normalized metric $\frac{\mu-\mu_{\min }}{\mu_{\max }-\mu_{\min }}$ for different values of $P_{\max }$ for 2 contending users, $\sigma^{2}=-110 \mathrm{dBm}$, and $\bar{\gamma}=10 \mathrm{~dB}$.

$m_{L+1}=\mu_{\max }$. The following theorem provides a complete characterization of an optimal power mapping.

Theorem 1: Let $m_{i}=\mu_{\min }+\left(\frac{\mu_{\max }-\mu_{\min }}{L+1}\right) i, 0 \leq i \leq$ $L+1$. Then a power mapping that sets

$$
\pi(\mu)=q_{i}, \quad \text { if } m_{i} \leq \mu<m_{i+1},
$$

optimizes the probability of success in (2). The corresponding optimal probability of success is

$$
P_{\text {succ }}^{\pi}=1-\frac{1}{L+1} .
$$

Proof: The proof is given in Appendix B.

In other words, the optimal support consists of equal length intervals: $m_{i+1}-m_{i}=m_{i}-m_{i-1}$, for $1 \leq i \leq L$. A larger dynamic range in the receive power allows more levels (larger $L$ ), which increases the success probability by improving the odds that the best user's signal can be resolved.

The following corollary generalizes Theorem 1 to metrics with arbitrary (non-uniform) continuous probability distributions.

Corollary 1: Let the metric $\mu$ be a continuous RV in the range $\left[\mu_{\min }, \mu_{\max }\right)$ with a monotonically increasing cumulative distribution function (CDF) $F(\mu)$. Then, the mapping

$$
\pi(\mu)=q_{i}, \quad \text { if } \quad \frac{i}{L+1} \leq F(\mu)<\frac{i+1}{L+1},
$$

for $0 \leq i \leq L+1$, maximizes the probability of success in (2).

Proof: The proof easily follows from Theorem 1 and the following two observations: (i) the transformation $G=F(\mu)$ creates a continuous RV $G$ that is uniformly distributed in the range $[0,1)$ regardless of the probability distribution of $\mu$, and (ii) a one-to-one mapping exists between $\mu$ and $F(\mu)$ since $F(\mu)$ is monotonically increasing.

To guarantee that the transmission by a single node in a time slot can be decoded properly, we set $P_{\min }=\sigma^{2} \bar{\gamma}$ henceforth. The optimal power mapping function and its dependence on the dynamic range $P_{\min }$ and $P_{\max }$ is illustrated in Fig. 1 when the metric is uniformly distributed. 


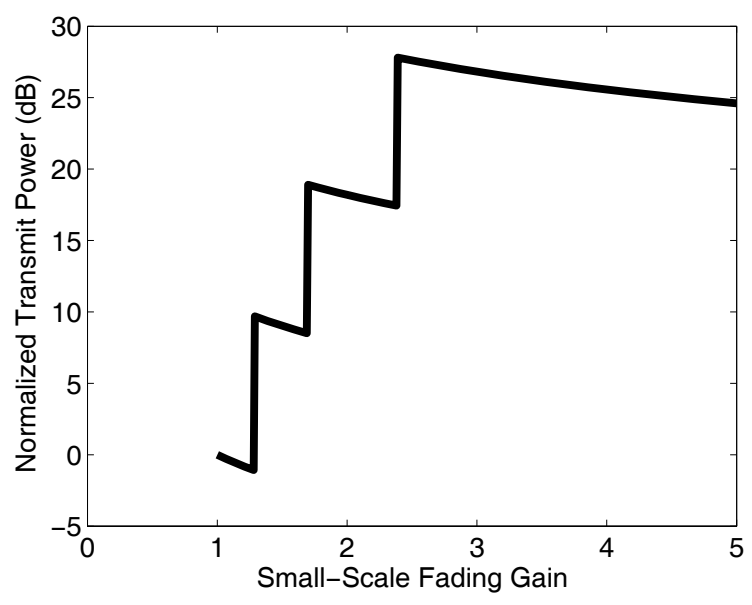

Fig. 2. The transmit power normalized with respect to $P_{\min }$ as a function of small-scale fading gain, $\alpha_{i}$, for $\mu_{\min }=1$ and $\mu_{\max }=\infty$.

\section{B. Metric-to-Transmit Power Mapping Examples}

We choose a specific example in multiuser diversity [32] to illustrate how the metric, receive power, and transmit power are related. Specifically, the channel (power) gain of a user $i$ can be written as $h_{i}=\bar{h}_{i} \alpha_{i}$, where $\bar{h}_{i}$ includes path loss, shadowing, and antenna gain, and $\alpha_{i}$ is due to shortterm Rayleigh fading with unity mean value. We choose the proportional-fair metric $\mu=h_{i} / \bar{h}_{i}=\alpha_{i}$, which is an exponential distribution function with unit mean, and its CDF is given by $F(\mu)=1-e^{-\mu}, 0 \leq \mu<\infty$. Lemma 1 shows how the receive power may be selected if exactly two nodes transmit, and the corresponding transmit power of user $i$ equals to $\pi\left(\alpha_{i}\right) / h_{i}$. In Fig. 2, the transmit power normalized with respect to $P_{\min }$ (i.e., $\pi\left(\alpha_{i}\right) /\left(P_{\min } \alpha_{i}\right)$ ) is plotted against $h_{i}$. It can be seen that the transmit power also ends up being discontinuous. But, unlike receive power, it is not an MND function of the metric.

\section{Simultaneous Transmissions from Exactly $n+1$ Nodes}

In general, when $n+1$ nodes transmit simultaneously, Lemma 3 can be generalized to show that the optimal power mapping still consists of discrete power levels. The levels are again determined iteratively starting from one level $q_{0}=P_{\min }$. Given a set of levels that have been computed thus far, each possible combination of $n$ of these levels leads to a possibly new and higher power level that can overcome the interference from the $n$ interferers.

To compute the receive power levels, we begin by defining a set $\mathbf{Q}_{0}=\left\{P_{\min }\right\}$, and we create a set $\mathbf{Q}_{k+1}$ based on the elements in the set $\mathbf{Q}_{k}$. Let $\Omega_{k}$ denote the set of all possible sets of $n$ levels chosen from $\mathbf{Q}_{k}$ that the $n$ nodes can occupy. Then,

$$
\mathbf{Q}_{k+1}=\mathbf{Q}_{k} \cup\left\{q: q=\bar{\gamma}\left(\sum_{x \in \omega} x+\sigma^{2}\right)<P_{\max }, \omega \in \Omega_{k}\right\}
$$

This procedure is repeated until no new power level gets added to the set. It can be easily shown that the iteration terminates.

The iteration above thus leads to a large number of levels and becomes intractable even for small $n$. We, therefore, derive a sub-optimal power mapping for this case that consists of fewer carefully designed power levels.

1) Overcoming worst-case interference from $n$ other nodes: The levels are set so as to ensure that the best user can be successfully captured even in the worst-case interference scenario in which the power received from each of the other $n$ contending nodes (adversaries) is just one level below the receive power of the best user.

In this case, the power levels are given by

$$
q_{n, i}=\bar{\gamma}\left(n q_{n, i-1}+\sigma^{2}\right), \quad i \in\left\{1,2, \cdots, L_{n}\right\},
$$

where $q_{n, 0}=P_{\min }$, and $L_{n}$ is the index of the largest power level. Solving for $q_{n, i}$ explicitly, we obtain

$$
q_{n, i}=(n \bar{\gamma})^{i} P_{\min }+\sigma^{2} \bar{\gamma} \frac{(n \bar{\gamma})^{i}-1}{n \bar{\gamma}-1}, \quad i \in\left\{0,1, \cdots, L_{n}\right\} .
$$

Using the maximum power constraint, it follows that

$$
L_{n}=\left\lfloor\log _{n \bar{\gamma}}\left(\frac{(n \bar{\gamma}-1) P_{\max }+\sigma^{2} \bar{\gamma}}{(n \bar{\gamma}-1) P_{\min }+\sigma^{2} \bar{\gamma}}\right)\right\rfloor .
$$

Note that setting $n=1$ leads to (5).

As in Theorem 1, we can optimize the support to maximize the probability of successfully capturing the best user. Recall that metrics in the range $\left[m_{i}, m_{i+1}\right)$ are mapped to receive power $q_{i}$, for $0 \leq i \leq L_{n}$, and $m_{0}=\mu_{\min }$ and $m_{L_{n+1}}=$ $\mu_{\max }$. Then, the probability of success, when exactly $n+1$ nodes are present, can be written in closed-form as follows:

$$
\begin{aligned}
P_{\text {succ }}^{n} & =\sum_{i=1}^{L_{n}} \operatorname{Pr}\left(\begin{array}{l}
\text { one metric lies in }\left[m_{i}, m_{i+1}\right), \\
\text { other } n \text { metrics are less than } m_{i}
\end{array}\right), \\
& =\frac{\sum_{i=1}^{L_{n}}\left(\begin{array}{c}
n+1 \\
1
\end{array}\right)\left(m_{i+1}-m_{i}\right)\left(m_{i}-m_{0}\right)^{n}}{\left(\mu_{\max }-\mu_{\min }\right)^{L_{n}+1}} .
\end{aligned}
$$

As before, the support can be optimized to maximize the probability of success. The following lemma characterizes the optimal support that maximizes $P_{\text {succ }}^{n}$.

Lemma 4: When the metric is uniformly distributed in $\left[\mu_{\min }, \mu_{\max }\right)$, the support

$$
m_{i}=\mu_{\min }+\left(\mu_{\max }-\mu_{\min }\right) \prod_{j=i}^{L_{n}} t_{j},
$$

for $i \in\left\{1,2, \cdots, L_{n}\right\}$, where $t_{1}=\frac{n}{n+1}$ and $t_{i}=\frac{n}{n+1-t_{i-1}^{n}}$, maximizes $P_{\text {succ }}^{n}$ in (14).

Proof: See Appendix C.

Figure 3 shows the optimal support for various $n$ and $L$ values, assuming the worst-case interference discussed above. (The largest value of $L$ depends on $P_{\min }$ and $P_{\max }$.) Notice that for $n=1$, the worst case interference is the same as interference from the node with the lower metric value. Thus, the solution reduces to the equal support case of Theorem 1.

\section{Interference from an unknown number of interferers}

In the previous section, the power levels were set so as to successfully overcome the interference from $n$ adversaries. During multiple access, the actual number of nodes that transmit in a slot is, in general, a random variable that takes 


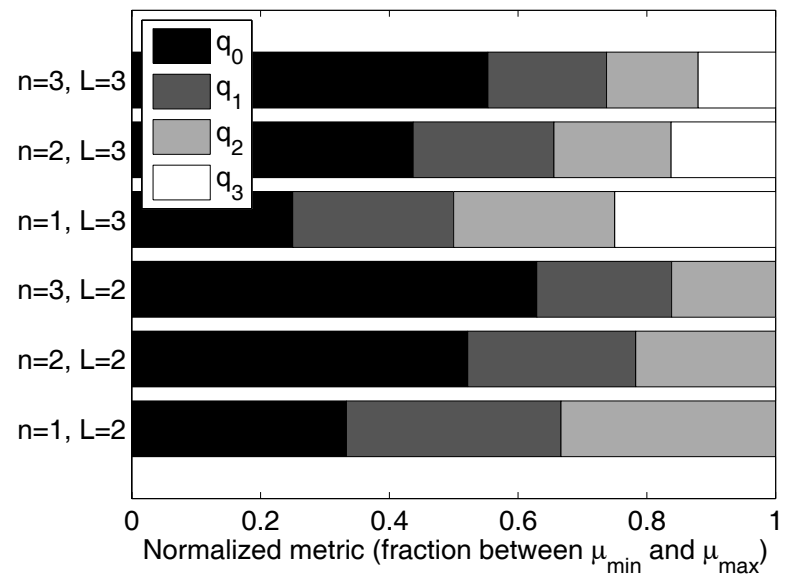

Fig. 3. The support for the metric-to-received-power mapping for different values of $L$ and $n$, as a function of the normalized metric $\frac{\mu-\mu_{\min }}{\mu_{\max }-\mu_{\min }}$.

value between 0 and $N$. In this paper, we set the power levels as follows:

$$
q_{a, i}=(a \bar{\gamma})^{i} P_{\min }+\sigma^{2} \bar{\gamma} \frac{(a \bar{\gamma})^{i}-1}{a \bar{\gamma}-1}, \quad 0 \leq i \leq L_{a},
$$

where $a \geq 1, a \in \mathbb{R}$, is called the adversary order. The number of power levels $L_{a}$ depends on $P_{\max }$, and the levels are set according to an adversary order $a$. A node with metric $\mu$ ensures that the power received from it is according to the following power mapping:

$$
\pi(\mu)=q_{a, i}, \quad \text { if } m_{i} \leq \mu<m_{i+1}, \quad 0 \leq i \leq L_{a},
$$

where the $\left\{m_{0}, \ldots, m_{L_{a}+1}\right\}$ is the support.

This ensures that the best node will always be captured when at most $\lfloor a\rfloor+1$ nodes transmit. This, therefore, leads to the following lower bound for the probability of success, $S_{r}$, when $r$ nodes actually transmit, for a uniform distribution of the metric:

$$
\begin{aligned}
S_{r} & = \begin{cases}\frac{r \sum_{i=1}^{L_{a}}\left(m_{i+1}-m_{i}\right)\left(m_{i}-m_{0}\right)^{r-1}}{\left(\mu_{\max }-\mu_{\min }\right)^{r}}, & 1 \leq r \leq\lfloor a\rfloor+1 \\
0, & r=0, r>\lfloor a\rfloor+1\end{cases} \\
& =\left\{\begin{array}{ll}
r \sum_{i=1}^{L_{a}}\left(T_{i+1}-T_{i}\right) T_{i}^{r-1}, & 1 \leq r \leq\lfloor a\rfloor+1 \\
0, & r=0, r>\lfloor a\rfloor+1
\end{array},\right.
\end{aligned}
$$

where $T_{i}=\frac{i}{L_{a}+1}$ for equal level intervals (based on Theorem 1), or $T_{i}=\prod_{j=1}^{L_{a}} t_{j}$ (based on Lemma 4) if one chooses to optimize the support for a specific number of transmitting nodes. This is a lower bound since it pessimistically assumes that capture never occurs when the number of transmitting nodes exceeds $\lfloor a\rfloor+1$. As we shall see in Sec. V, this bound is quite tight. Henceforth, specifying the adversary order and the support will fully define the power mapping function.

There are several interesting trade-offs that occur in choosing the appropriate adversary order $a$. Increasing $a$ increases the gap between the power levels, and, thus, improves the odds of capture. But, it also may reduce the number of levels $L_{a}$ (since the receive power levels must lie between $P_{\min }$ and $\left.P_{\max }\right)$, which instead increases the probability of no capture. Another trade-off occurs in determining the support. While optimizing the support for $\lfloor a\rfloor+1$ contending nodes, as per Sec. III-C, results in the best probability of success when exactly $\lfloor a\rfloor+1$ nodes transmit, it decreases the probability of success when fewer nodes transmit. Therefore, it makes sense to decouple the optimization of the support from the optimization of $a$. These trade-offs play an important role in the design of the overall MAS algorithm in the next section.

\section{Variable Power Multiple Access Selection ALGORITHM}

We now design the Variable Power Multiple Access Selection (VP-MAS) algorithm that controls: (i) how many nodes transmit at any time so as to improve the odds of capturing the best user, and (ii) what power mapping function (defined in terms of the adversary order and the support) to use for the nodes that do transmit. Intuitively, controlling the number of nodes that transmit is important because more steps will be required to find the best user if too few users transmit in each step. On the other hand, if too many users simultaneously transmit, the interference increases and reduces the odds of a successful capture. The power mapping matters because the success probability suffers significantly when more users transmit than can be handled by the adversary-order based setting of the power levels. On the other hand, assuming a pessimistically large adversary order is also not desirable since it decreases the number of power levels available.

The MAS algorithm proceeds through a sequence of steps and eventually results in the successful capture of the signal sent by the best user. In each step, only nodes whose metrics lie within a specified interval determined by the algorithm transmit. At the end of each step, the base station broadcasts one of three outcomes to all nodes: idle, collision, or success. Depending on the outcome, the intervals are updated, as described below. This updating can be done independently by each node without any additional feedback from the sink.

The development of the VP-MAS algorithm below limits itself to metrics uniformly distributed in $[0,1)$ in order to make it approachable and simple. Appendix D shows how this can be fully generalized to the case in which the metric has any monotonically increasing CDF.

Definitions: To specify the protocol precisely and optimize its performance, we first define the following three state variables: $\mu_{\text {base }}(k), \mu_{\max }(k)$, and $\mu_{\min }(k) . \mu_{\text {base }}(k)$ and $\mu_{\max }$ are the lowest and highest possible values, respectively, of the best metric at the beginning of step $k$. In step $k$, only nodes with metrics between $\mu_{\min }(k)$ and $\mu_{\max }(k)$ transmit. Hence, if equal length support intervals are used, the supports at step $k$ that determine the receive power level are

$m_{i}=\mu_{\min }(k)+T_{i}\left(\mu_{\max }(k)-\mu_{\min }(k)\right), \quad$ for $0 \leq i \leq L+1$,

where $T_{i}$ takes on the same meaning as in (18). We also define $z(k)$ as the probability that an arbitrary node will transmit in step $k$. Let $\varphi(k)$ denote the most likely estimate of the number of nodes with metrics between $\mu_{\text {base }}(k)$ and $\mu_{\max }(k)$.

Initialization: At the beginning of the algorithm, the best metric can lie anywhere between $\mu_{\min }$ and $\mu_{\max }$. Therefore, $\mu_{\text {base }}(1)=0$ and $\mu_{\max }(1)=1$. Initially, all $N$ nodes lie between $\mu_{\text {base }}(1)$ and $\mu_{\max }(1)$. Therefore, $\varphi(1)=N$. (Given 
these initial values, $z(1)$ gets automatically determined by (21) given below.)

Basic relationships: Given $\varphi(k)$ and $z(k)$, the success probability in step $k$ is lower bounded by

$$
P^{\text {succ }}(k)=\sum_{r=1}^{\varphi(k)} S_{r}\left(\begin{array}{c}
\varphi(k) \\
r
\end{array}\right) z(k)^{r}(1-z(k))^{\varphi(k)-r},
$$

where $S_{r}$, given by (18), is the probability of success when $r$ nodes transmit. Note that $S_{r}$ only depends on the adversary order $a$, and how the system chooses to compute $\left\{T_{i}\right\}_{i=1}^{L_{a}}$.

The key rule is that the parameters are updated so as to maximize the probability of success in each step. To achieve this, it follows from (20), that the transmission probability, $z(k)$, needs to be set as:

$$
z(k)=\arg \max _{0 \leq \varepsilon \leq 1} \sum_{r=1}^{\varphi(k)} S_{r}\left(\begin{array}{c}
\varphi(k) \\
r
\end{array}\right) \varepsilon^{r}(1-\varepsilon)^{\varphi(k)-r} .
$$

Given that all nodes with metrics that lie between $\mu_{\min }(k)$ and $\mu_{\max }(k)$ transmit, $z(k)$ can be written in terms of the state variables as $z(k)=\frac{\mu_{\max }(k)-\mu_{\min }(k)}{\mu_{\max }(k)-\mu_{\text {base }}(k)}$. Therefore,

$$
\mu_{\min }(k)=\mu_{\max }(k)-\left(\mu_{\max }(k)-\mu_{\text {base }}(k)\right) z(k) .
$$

VP-MAS Algorithm: At each time slot $k$, VP-MAS proceeds as follows:

1) A node $i$ with metric $\mu_{i}$ transmits only if $\mu_{i}$ lies in the range $\left[\mu_{\min }(k), \mu_{\max }(k)\right)$. If it transmits, the power mapping it uses, $\pi\left(\mu_{i}\right)$, is calculated from (17) and (19).

2) If the outcome is a success, then the best node has been captured and the algorithm terminates.

3 ) If the outcome is an idle, then no node transmitted. This implies that all the nodes, including the best node, have metrics that are less than $\mu_{\min }(k)$ (and greater than $\left.\mu_{\text {base }}(k)\right)$. Hence, $\mu_{\max }(k+1)=\mu_{\min }(k)$ and $\mu_{\text {base }}(k+1)=\mu_{\text {base }}(k)$. Thus, the estimate of the number of nodes lie between $\mu_{\max }(k+1)$ and $\mu_{\text {base }}(k+1)$ remains unchanged. Hence, $\varphi(k+1)=\varphi(k)$. Therefore, from (21), it also follows that $z(k+1)=z(k)$. $\mu_{\min }(k+1)$ follows from (22).

4) If the outcome is a collision, it implies that the best metric definitely lies between $\mu_{\min }(k)$ and $\mu_{\max }(k)$. Hence, we set $\mu_{\max }(k+1)=\mu_{\max }(k)$ and $\mu_{\text {base }}(k+$ $1)=\mu_{\min }(k)$. Furthermore, $\varphi(k+1)$, the most likely number of nodes between $\mu_{\max }(k+1)$ and $\mu_{\text {base }}(k+1)$, is

$$
\begin{gathered}
\varphi(k+1)= \\
\arg \max _{2 \leq r \leq \varphi(k)}\left(\begin{array}{c}
\varphi(k) \\
r
\end{array}\right) z(k)^{r}(1-z(k))^{\varphi(k)-r}\left(1-S_{r}\right) .
\end{gathered}
$$

The values of $z(k+1)$, and, consequently, $\mu_{\min }(k+1)$, are determined using (21) and (22).

Example: We demonstrate all the possibilities of the algorithm using the following contrived example with $N=6$ nodes, $\bar{\gamma}=10 \mathrm{~dB}, \sigma^{2}=-110 \mathrm{dBm}$, and $P_{\max }=-70 \mathrm{dBm}$. The optimal receive power levels are then given by $-100 \mathrm{dBm}$, $-84.9 \mathrm{dBm}$, and $-70 \mathrm{dBm}$. The metrics of nodes 1 to 6 are assumed to be $0.1548,0.2731,0.4324,0.5749,0.6440$, and
0.7011 , respectively. In slot 1 , with $\mu_{\text {base }}(1)=0, \mu_{\max }(1)=1$, and $\varphi(1)=6$, the algorithm computes $\mu_{\min }(1)=0.71$. Since none of the metrics exceeds 0.71 , an idle slot results. The algorithm then sets $\mu_{\text {base }}(2)=0, \mu_{\max }(2)=0.71$, and $\varphi(2)=6$, which leads to $\mu_{\min }(2)=0.5041$. This implies that the mapping function in slot 2 maps metrics in $[0.5041,0.5727)$ to $-100 \mathrm{dBm}$, in $[0.5727,0.6414)$ to $-84.9 \mathrm{dBm}$, and in $[0.6414,0.71)$ to $-70 \mathrm{dBm}$ receive power. Hence, the receive powers from nodes 4,5 and 6 are $-100 \mathrm{dBm},-70 \mathrm{dBm}$, and $-70 \mathrm{dBm}$, respectively, which leads to a collision. The algorithm then sets $\mu_{\text {base }}(3)=0.5041$ and $\mu_{\max }(3)=0.71$, and computes $\varphi(3)=2$ and $\mu_{\min }(3)=$ 0.5556 . This implies that the mapping function in slot 3 maps metrics in $[0.5556,0.6071)$ to $-100 \mathrm{dBm}$, in $[0.6071,0.6585)$ to $-84.9 \mathrm{dBm}$, and in $[0.6585,0.71)$ to $-70 \mathrm{dBm}$ receive power. Now, the receive powers from nodes 4,5 and 6 are $-100 \mathrm{dBm}$, $-84.9 \mathrm{dBm}$, and $-70 \mathrm{dBm}$, respectively, which leads to the desired successful capture of best node 6's packet.

\section{A. VP-MAS with Power-Based Splitting (VP-MAS-PS)}

We now develop a more efficient variant of VP-MAS that leverages the ability, if present, of the receiver to measure the total receive power. Most receiver implementations already support this capability since they measure received signal strength indicator (RSSI). This capability was not required of the receiver in VP-MAS, which was developed in the previous section.

Such a capability is especially useful in the event of a collision as it indicates the interval in which the maximum metric value lies. This is so because the gap between adjacent power levels given by $\pi(\cdot)$, shown in (17), increases exponentially with $a \bar{\gamma}$. Therefore, the signal from the power level that is the highest among the ones selected by the users, comprises the bulk of the received signal power. For example, in lightly coded systems, the SINR threshold, $\bar{\gamma}$, is in the order of 6$15 \mathrm{~dB}$, which implies that $a \bar{\gamma}>4$. Therefore, 4 or more interferers will need to transmit with the same receive power for the receiver to mistakenly infer that a single node (with higher receive power) transmitted. This is highly unlikely.

The receiver can therefore assume that, with high probability, the total receive power in step $k, P^{t o t}(k)$, consists of at least one node whose receive power is at the highest level below $P^{t o t}(k)$. Hence, it inverts the power mapping to determine the support interval in which the best user's metric lies. This information determines which users transmit in the next step. Formally, the receiver assumes that the best node's metric lies in the range $\left[m_{J(k)}, m_{J(k)+1}\right)$, where $J(k)$ is the estimate of the receive level of the best user. It is chosen as the level that is closest to $P^{t o t}(k)$ :

$$
J(k)=\max \left\{\begin{array}{l}
0 \leq i \leq L_{a}: \\
(a \bar{\gamma})^{i} P_{\min }+\sigma^{2} \bar{\gamma} \frac{(a \bar{\gamma})^{i}-1}{a \bar{\gamma}-1} \leq P^{t o t}(k)
\end{array}\right\} .
$$

While the above estimate is good, it need not always be correct. For example, the estimate can be too high when many nodes simultaneously transmit. In this case, no node will end up transmitting and $J(k+1)$ will need to be lower than $J(k)$. Therefore, for VP-MAC-PS, the response to an idle 
outcome differs depending on whether a collision has occurred previously or not. If no collision has occurred previously, the idle outcome is handled in the same way as VP-MAS. However, if a collision has previously occurred, it implies that $J(k)$ is too high. The base station then decrements $J(k)$ in the next step, and broadcasts it as well.

VP-MAS-PS maintains two state variables $\mu_{\min }(k)$ and $\mu_{\max }(k)$, which are initialized just as in VP-MAS. In addition, it calculates $\varphi(k), z(k)$, and $J(k)$. Given that the receiver assumes that the best metric lies between $\left[m_{J(k)}, m_{J(k)+1}\right)$, $\mu_{\text {base }}(k)$ is no longer useful and is set to 0 .

At each time slot $k$, VP-MAC-PS thus proceeds as follows for uniformly distributed metrics. The non-uniform metric case is handled in Appendix D.

1) A node $i$ with metric $\mu_{i}$ transmits if $\mu_{i} \in$ $\left[\mu_{\min }(k), \mu_{\max }(k)\right)$, such that its receive power, $\pi\left(\mu_{i}\right)$, is as per (17). The support, $\left\{m_{0}, \ldots, m_{L_{a}+1}\right\}$, is updated just as in VP-MAS as a function of $\mu_{\min }(k)$ and $\mu_{\max }(k)$.

2) If the outcome is a success, the algorithm terminates.

3 ) If the outcome is a collision, the receiver computes the estimate $J(k)$ from the total receive power $P^{t o t}(k)$ using (24), and broadcasts it to all nodes as its estimate of the level of the best node. Consequently, in the case of equal length interval support,

$$
\mu_{\min }(k+1)=\mu_{\min }(k)+\frac{\mu_{\max }(k)-\mu_{\min }(k)}{L_{a}+1} J(k),
$$

and

$$
\mu_{\max }(k+1)=\mu_{\min }(k)+\frac{\mu_{\max }(k)-\mu_{\min }(k)}{L_{a}+1}(J(k)+1) .
$$

4) If the outcome is idle and no collision has occurred so far, $\mu_{\max }(k+1)=\mu_{\min }(k), \varphi(k+1)=N$, and $z(k+1)=z(k)$. As in VP-MAS, $\mu_{\min }(k+1)$ is calculated using (22).

5) If the outcome is idle and a collision has occurred previously, the receiver also broadcasts $J(k)=J(k-1)-1$. The most likely range in which the maximum metric resides is then the metric value corresponding to the index that is one less than the previous estimate. Hence, $\mu_{\max }(k+1)=\mu_{\min }(k)$, and $\mu_{\min }(k+1)=\mu_{\min }(k)-$ $\left(\mu_{\max }(k)-\mu_{\min }(k)\right)=2 \mu_{\min }(k)-\mu_{\max }(k)$.

\section{Simulations}

We consider a network of multiple transmitting nodes and a common information sink. Each node has a metric that is uniformly distributed in the range $[0,1)$. The metrics are independent across different nodes. We assume that a packet is decoded successfully when its SINR exceeds $\bar{\gamma}=10 \mathrm{~dB}$. Furthermore, we assume the effective noise floor has a power $\sigma^{2}=-110 \mathrm{dBm}$. Hence, $P_{\min }=\sigma^{2} \bar{\gamma}=-100 \mathrm{dBm}$. In the simulations, we test the performance of the system when the maximum receive power $P_{\max }$ is $-80,-70$, and $-60 \mathrm{dBm}$. For each set of parameters, data is collected over 100,000 independent trials.

We first examine how the adversary order, $a$, and the support affect VP-MAS. Figure 4 plots, for 3 values of $P_{\max }$, the

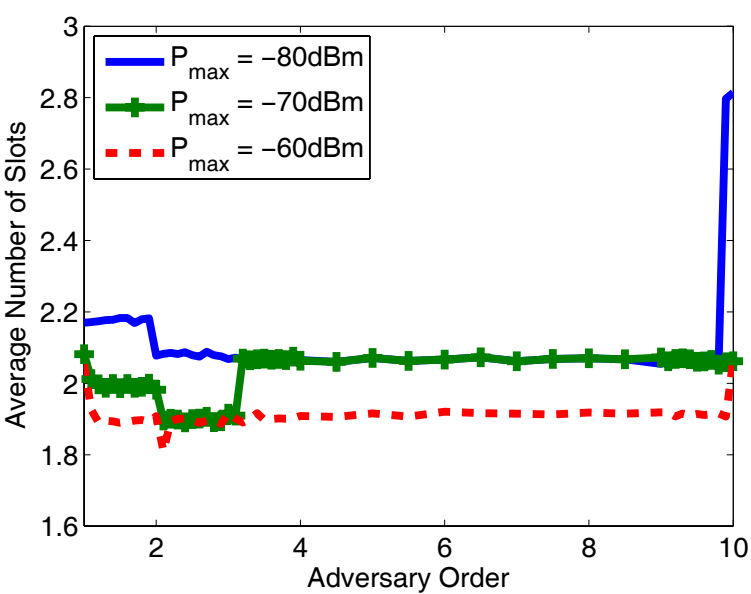

Fig. 4. Average number of slots required to find the node with the best metric using VP-MAS as a function of adversary order, $a$, when the support is optimized for simultaneous transmissions by 2 users. $(N=50$ nodes, $\sigma^{2}=-110 \mathrm{dBm}$, and $\bar{\gamma}=10 \mathrm{~dB}$ )

average number of slots required to select the best user among $N=50$ users. As mentioned, as $a$ increases, the gap between the discrete power levels also increases. This initially improves the probability of capture and reduces the average number of slots required. This effect is particularly prominent when $a$ goes from below 2 to above 2 , since $a=2.1$ means that the packet from the best node can still be decoded even when the receive powers of two major interferers are at the level immediately below it, and other interferers are at a much lower received power. On the other hand, when $a=1.9$, for example, the packet from the best node can be decoded in the presence of at most one major interferer at a level below it. However, increasing $a$ further eventually leads to a reduction in the number of levels. It is this reduction in the number of levels that dramatically worsens the performance. For example, when $P_{\max }=-80 \mathrm{dBm}$, the number of levels supported is 2 for $a<9.9$, and it decreases to 1 for larger $a$. Therefore, the average number of slots required to select the best user increases from 2.1 to 2.8 when $a$ goes beyond 9.9. This same result causes the performance degradation at $a=3.2$ for $P_{\max }=-70 \mathrm{dBm}$, and at $a=2.2$ for $P_{\max }=-60 \mathrm{dBm}$. Larger values of $a$ lead to negligible performance gains since a large number of interferers seldom simultaneously transmit in VP-MAS. When $P_{\max }=-60 \mathrm{dBm}$, the lowest average number of slots is just 1.8. This is a remarkably low number since at least one slot is always required to select the best user.

Figure 5 plots the performance of VP-MAS when the support is optimized to best handle 3 simultaneous transmissions, as opposed to 2 simultaneous transmissions in the previous figure. We can see that increasing $n$ from 1 to 2 has a negligible effect of performance. In some cases, e.g., when $P_{\max }=-60 \mathrm{dBm}$ and $a=2.1$, the average number of slots does decrease to 1.75 . However, the performance otherwise is very similar to the equal support case. Therefore, we shall henceforth use equal interval supports. Doing so has the advantage of enabling the supports to be calculated in a closed and simple form very easily from Theorem 1 . This avoids 


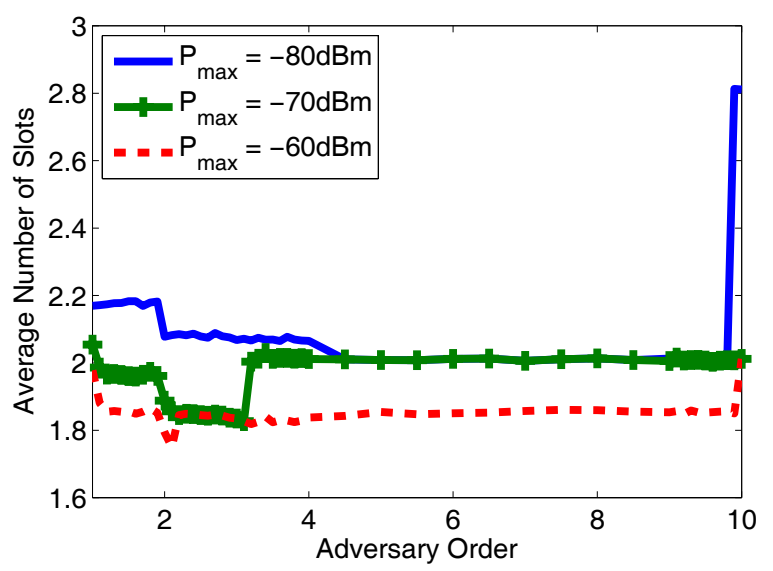

Fig. 5. Average number of slots required by VP-MAS to select the best node as a function of adversary order, $a$, when the support is optimized for simultaneous transmissions by 3 users. $\left(N=50\right.$ nodes, $\sigma^{2}=-110 \mathrm{dBm}$, and $\bar{\gamma}=10 \mathrm{~dB}$ )

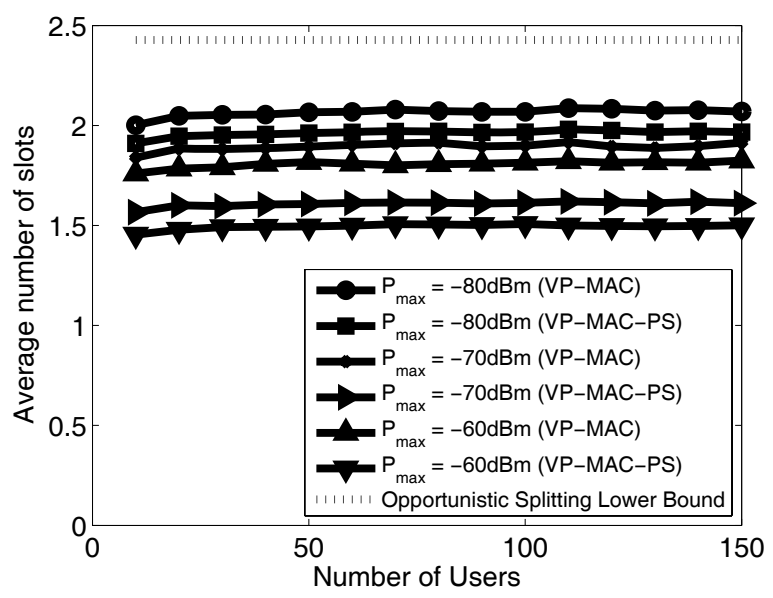

Fig. 6. Average number of slots required to select the best node with the best metric using VP-MAS and VP-MAS-PS algorithms $\left(\sigma^{2}=-110 \mathrm{dBm}\right.$, $\bar{\gamma}=10 \mathrm{~dB}$ ). Also shown is the 2.43 slot lower bound when only collision is assumed.

the more involved recursion of Lemma 4 in each step of the algorithm every time $\mu_{\min }(k)$ and $\mu_{\max }(k)$ are updated.

Using the above parameters, we now vary the number of nodes in the network from 10 to 150 in Figure 6. It can be seen that even with many users, $2.1,1.9$, and 1.8 slots are required for $P_{\max }=-80 \mathrm{dBm},-70 \mathrm{dBm}$, and $-60 \mathrm{dBm}$, respectively. For VP-MAS-PS, the numbers further reduce to requires 2.0, 1.6 and 1.5 slots, respectively. Also plotted in the figure is the 2.43 slot lower bound on the average number of slots when the standard collision model is assumed [19]. Thus, using just one extra power level, VP-MAS shaves off 0.5 slots. And, using two levels, it shaves off 1.0 slots, which is a significant gain. Note that the average number of slots is insensitive to the number of nodes, $N$, in the network. This happens because VP-MAS adjusts $\mu_{\min }(1)$ according to the number of nodes and the probability distribution of the metric to limit the number of nodes that contend in each step of the algorithm.

Figure 7 plots the probability of a successful capture as

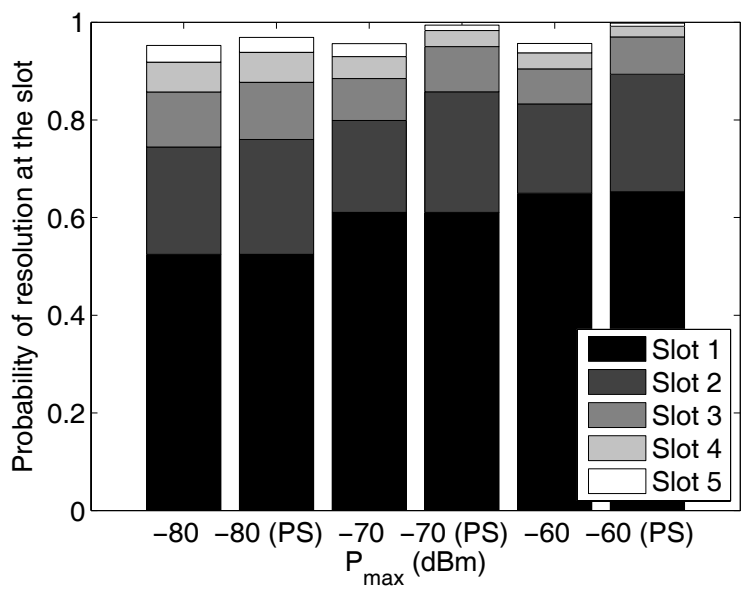

Fig. 7. Probability of finding the best user as a function of the steps (slots) for VP-MAS and VP-MAS-PS $\left(N=50, \sigma^{2}=-110 \mathrm{dBm}\right.$, and $\left.\bar{\gamma}=10 \mathrm{~dB}\right)$.

a function of the number of steps (slots) into the algorithm, and explains why VP-MAS and VP-MAS-PS are this efficient. We can see that the asymmetric receive power levels enable the algorithms to readily capture the best user more than half the time in the first slot itself. While both VP-MAS and VP-MAS-PS have the same probability of success in the first slot, the probability of capture in subsequent slots is greater for the VP-MAS-PS. For example, for VP-MAS, the probability of success in the first slot increases from $53.5 \%$ at $P_{\max }=-80 \mathrm{dBm}(20 \mathrm{~dB}$ dynamic range $)$ to $65.0 \%$ at $P_{\max }=-60 \mathrm{dBm}(40 \mathrm{~dB}$ dynamic range).

\section{CONCLUSIONS}

In this paper, we considered the problem of contentionbased multiple access selection in which the goal is to find the user with the best metric (e.g., best channel) by successfully receiving its signal first. We showed that multiple access behavior changes significantly when we take into account local channel state information, capture, and power control. The best strategy is to transmit in such a way that the receive power falls into one of a set of discrete levels. We optimized those levels and the mapping of the metric onto them. Based on these results we developed the VP-MAS algorithm that allows effective multiple access selection by dynamically adjusting the power levels depending on whether previous transmission attempts resulted in capture, idle channels, or collisions. We also developed a faster variant called the VP-MAS-PS algorithm that exploits received signal strength information at the receiver, should it be available. The results of this paper can be used for more effective and faster random access in systems with multiuser diversity, which is important for highspeed data transmission, as well as for the association and setup phase in many cellular systems. This work also provides a mechanism for relay selection in cooperative communication systems, which is much more efficient and scalable than centralized polling mechanisms.

We believe that this paper opens up several avenues for future work. For example, even faster mechanisms may be possible when the system is interested not in the best node, but in any one of the many nodes that meet a certain criterion. 
Another extension is the case where the system parameters or statistics are not known perfectly.

\section{APPENDIX}

\section{A. Proof of Lemma 3}

Let $\mu_{a}$ and $\mu_{b}$ be an ordering of uniformly distributed random variables such that $\mu_{a} \leq \mu_{b}$, and let $\delta=\mu_{b}-\mu_{a}$. From order statistics, the pdfs of $\mu_{a}$ and $\delta$ conditioned on $\mu_{a}$ can be written as [33]:

$$
\begin{aligned}
p\left(\mu_{a}\right) & =\frac{2\left(\mu_{\max }-\mu_{a}\right)}{\left(\mu_{\max }-\mu_{\min }\right)^{2}}, \quad \mu_{\min } \leq \mu_{a} \leq \mu_{\max } \\
p\left(\delta \mid \mu_{a}\right) & =\frac{1}{\mu_{\max }-\mu_{a}}, \quad 0 \leq \delta \leq \mu_{\max }-\mu_{a} .
\end{aligned}
$$

For any power mapping $\pi($.$) , a successful capture occurs only$ if $\frac{\pi\left(\mu_{a}+\delta\right)}{\pi\left(\mu_{a}\right)+\sigma^{2}} \geq \bar{\gamma}$.

Let $\pi^{\text {inv }}(x)$ denote the smallest value of $\mu$ at which $\pi(\mu) \geq x$. (Since $\pi($.$) is \operatorname{MND}, \pi(\mu) \geq x$ for all $\mu \geq$ $\pi^{\text {inv }}(x)$.) Therefore, for a given $\mu_{a}$, capture is successful for all $\delta \geq \pi^{\text {inv }}\left(\bar{\gamma} \pi\left(\mu_{a}\right)+\bar{\gamma} \sigma^{2}\right)-\mu_{a}$. Note that the above definitions hold even for a discontinuous (but MND) $\pi$.

For any (MND) power mapping $\pi$, the general expression for probability of success is:

$$
\begin{aligned}
P_{\mathrm{succ}}^{\pi} & =\int_{\mu_{\min }}^{\mu_{\max }} \int_{\xi}^{\mu_{\max }-\mu_{a}} \frac{2\left(\mu_{\max }-\mu_{a}\right)}{\left(\mu_{\max }-\mu_{\min }\right)^{2}} \frac{1}{\mu_{\max }-\mu_{a}} d \delta d \mu_{a} \\
& =\frac{2 \int_{\mu_{\min }}^{\mu_{\max }}\left[\mu_{\max }-\pi^{\mathrm{inv}}\left(\bar{\gamma} \pi\left(\mu_{a}\right)+\bar{\gamma} \sigma^{2}\right)\right]^{+} d \mu_{a}}{\left(\mu_{\max }-\mu_{\min }\right)^{2}}
\end{aligned}
$$

where $\xi=\min \left(\pi^{\mathrm{inv}}\left(\bar{\gamma} \pi\left(\mu_{a}\right)+\bar{\gamma} \sigma^{2}\right)-\mu_{a}, \mu_{\max }-\mu_{a}\right)$. We can assume that the optimal solution has $\pi\left(\mu_{\min }\right)=P_{\min } \cdot{ }^{7}$ If $\frac{P_{\max }}{P_{\min }+\sigma^{2}}<\bar{\gamma}$, then $P_{\text {succ }}^{\pi}$ is always 0 for any mapping. Otherwise, let $m_{1}$ be the smallest value at which $\pi\left(m_{1}\right) \geq q_{1}$, where $q_{1}=\bar{\gamma}\left(P_{\min }+\sigma^{2}\right)$, as defined in (6). (If $\pi($.$) does not have$ such a value, then its success probability is 0 . This implies that $\pi$ is necessarily suboptimal as a trivial modification of it such as setting $\pi(\mu)=P_{\max }$, for $\left(\mu_{\max }-\mu_{\min }\right) / 2 \leq \mu \leq \mu_{\max }$, results in a non-zero success probability.)

Consider an alternate mapping $\pi_{*}$ in which

$$
\pi_{*}(\mu)= \begin{cases}P_{\min }, & \mu_{\min } \leq \mu<m_{1} \\ \pi(\mu), & m_{1} \leq \mu \leq \mu_{\max }\end{cases}
$$

The probability of success of $\pi$ in (30) can be upper bounded

${ }^{7}$ Given any optimal solution, we can always construct a new mapping $\pi_{*}($. such that $\pi_{*}\left(\mu_{\min }\right)=P_{\min }$, and $\pi_{*}(\mu)=\pi(\mu)$ for all $\mu \in\left(\mu_{\min }, \mu_{\max }\right]$. Doing so guarantees that $\pi_{*}^{\text {inv }}\left(\bar{\gamma} \pi_{*}\left(\mu_{a}\right)+\bar{\gamma} \sigma^{2}\right) \leq \pi^{\text {inv }}\left(\bar{\gamma} \pi\left(\mu_{a}\right)+\bar{\gamma} \sigma^{2}\right)$, and thus ensures that $P_{\text {succ }}^{\pi_{*}} \geq P_{\text {succ }}^{\pi}$. as

$$
\begin{aligned}
P_{\text {succ }}^{\pi}= & \frac{2 \int_{\mu_{\min }}^{m_{1}}\left[\mu_{\max }-\pi^{\mathrm{inv}}\left(\bar{\gamma} \pi\left(\mu_{a}\right)+\bar{\gamma} \sigma^{2}\right)\right]^{+} d \mu_{a}}{\left(\mu_{\max }-\mu_{\min }\right)^{2}} \\
& +\frac{2 \int_{m_{1}}^{\mu_{\max }}\left[\mu_{\max }-\pi^{\mathrm{inv}}\left(\bar{\gamma} \pi\left(\mu_{a}\right)+\bar{\gamma} \sigma^{2}\right)\right]^{+} d \mu_{a}}{\left(\mu_{\max }-\mu_{\min }\right)^{2}} \\
\leq & \frac{2 \int_{\mu_{\min }}^{m_{1}}\left[\mu_{\max }-m_{1}\right]^{+} d \mu_{a}}{\left(\mu_{\max }-\mu_{\min }\right)^{2}} \\
& +\frac{2 \int_{m_{1}}^{\mu_{\max }}\left[\mu_{\max }-\pi_{*}^{\mathrm{inv}}\left(\bar{\gamma} \pi_{*}\left(\mu_{a}\right)+\bar{\gamma} \sigma^{2}\right)\right]^{+} d \mu_{a}}{\left(\mu_{\max }-\mu_{\min }\right)^{2}} \\
= & P_{\text {succ }}^{\pi_{*}},
\end{aligned}
$$

where $[x]^{+}=\max (x, 0)$. The inequality in (33) follows because $\pi$ is MND, which implies that $\pi^{\text {inv }}\left(\bar{\gamma} \pi(\mu)+\bar{\gamma} \sigma^{2}\right) \geq m_{1}+\bar{\gamma} \sigma^{2}$, for all $\mu_{\text {min }} \leq \mu<m_{1}$. Furthermore, from (31), $\pi_{*}^{\text {inv }}\left(\bar{\gamma} \pi_{*}(\mu)+\bar{\gamma} \sigma^{2}\right)=\pi^{\text {inv }}\left(\bar{\gamma} \pi(\mu)+\bar{\gamma} \sigma^{2}\right)$, for all $m_{1} \leq \mu \leq \mu_{\max }$. The inequality thus shows that a flat $\pi$ for $\mu \in\left[\mu_{\min }, m_{1}\right)$ has the highest success probability.

We now use the above argument successively to show that the optimal $\pi$ maps the metric values into a discrete set of power levels. Let $m_{0}=\mu_{\min }$. Assume that one has shown that the optimal $\pi$ maps metrics $\left[m_{i}, m_{i+1}\right)$ to power level $q_{i}$, for $0 \leq i \leq k$, for some $k \leq L$. The previous paragraph proved that the assumption is true for $k=0$. Define $q_{k+1}=$ $\bar{\gamma} q_{k}+\bar{\gamma} \sigma^{2}$, and $m_{k+1}=\pi^{\text {inv }}\left(q_{k+1}\right)$. If $q_{k+1} \leq P_{\max }$, the probability of success is

$$
\begin{aligned}
P_{\text {succ }}^{\pi} & =\sum_{j=0}^{k} \frac{2 \int_{m_{j}}^{m_{j+1}}\left[\mu_{\max }-m_{j}\right]^{+} d \mu_{a}}{\left(\mu_{\max }-\mu_{\min }\right)^{2}} \\
& +\frac{2 \int_{m_{k+1}}^{m_{k+2}}\left[\mu_{\max }-\pi^{\operatorname{inv}}\left(\bar{\gamma} \pi\left(\mu_{a}\right)+\bar{\gamma} \sigma^{2}\right)\right]^{+} d \mu_{a}}{\left(\mu_{\max }-\mu_{\min }\right)^{2}} \\
& +\frac{2 \int_{m_{k+2}}^{\mu_{\max }}\left[\mu_{\max }-\pi^{\mathrm{inv}}\left(\bar{\gamma} \pi\left(\mu_{a}\right)+\bar{\gamma} \sigma^{2}\right)\right]^{+} d \mu_{a}}{\left(\mu_{\max }-\mu_{\min }\right)^{2}} .
\end{aligned}
$$

As before, the middle term in the expression is upper bounded by $\frac{2}{\left(\mu_{\max }-\mu_{\min }\right)^{2}} \int_{m_{k+1}}^{m_{k+2}}\left[\mu_{\max }-m_{k+1}\right]^{+} d \mu_{a}$, with equality if $\pi$ maps $\left[m_{k}, m_{k+1}\right)$ to $q_{k}$. As before, this mapping does not affect the first and third term in the expression.

When $k=L$, it follows from (5) that $q_{k+1}>P_{\max }$. For this case, the probability expression has the same form as (35), except that it lacks the third term. Again, it can be argued that reducing all power levels to $q_{L}$ for $\mu \geq m_{L}$ does not affect $P_{\text {succ }}^{\pi}$. Hence, the result.

\section{B. Proof of Theorem 1}

From Lemma 3, we know that the optimal power mapping is discrete and consists of $L+1$ levels. Let $m_{0}=\mu_{\min }, m_{1}, \cdots, m_{L}, m_{L+1}=\mu_{\max }$ denote the support of the MND power mapping, so that $\pi(\mu)=q_{i}$ whenever $\mu \in\left[m_{i}, m_{i+1}\right)$, for $i \in\{0,1, \cdots, L\}$. The power levels are such that when $\mu_{a} \in\left[m_{j}, m_{j+1}\right)$, for some $j$, then the packet from $b$ can be decoded successfully for all $\mu_{b} \geq m_{j+1}$. 
Therefore, the probability of success expression in (29) can be simplified as follows:

$$
\begin{aligned}
P_{\text {succ }}^{\pi} & =\frac{2 \sum_{i=1}^{L+1} \int_{m_{i-1}}^{m_{i}} \int_{m_{i}-m}^{\mu_{\max }-m} d \delta d \mu_{a}}{\left(\mu_{\max }-\mu_{\min }\right)^{2}} \\
& =\frac{2 \sum_{i=1}^{L+1}\left(m_{i}-m_{i-1}\right)\left(\mu_{\max }-m_{i}\right)}{\left(\mu_{\max }-\mu_{\min }\right)^{2}} .
\end{aligned}
$$

The goal is to find the support $\left\{m_{i}\right\}_{i=1}^{L}$ that maximizes $P_{\text {succ }}^{\pi}$. By using the first order condition, it is easy to show that the optimal support is as given in the theorem statement.

\section{Proof of Lemma 4}

When we rearrange the first order condition of (14), we get the following recursion: $\frac{m_{1}-m_{0}}{m_{2}-m_{0}}=\frac{n}{n+1}$, and $\frac{m_{i}-m_{0}}{m_{i+1}-m_{0}}=$ $\frac{n}{n+1-\left(\frac{m_{i-1}-m_{0}}{m_{i}-m_{0}}\right)^{n}}, \quad i \in\left\{2,3, \cdots, L_{n}\right\}$. Let $t_{i}=\frac{m_{i}-m_{0}}{m_{i+1}-m_{0}}$. The recursion gives an analytical solution for $t_{L_{n}}$ in terms of $n$. It can be used to solve for $m_{L_{n}}$, since $m_{0}=\mu_{\min }$ and $m_{L_{n}+1}=\mu_{\max }$. Once $m_{L_{n}}$ is found, it can then be used to compute $m_{L_{n}-1}$, and so on. This leads to the desired formula.

\section{General VP-MAS for Metrics with Non-Uniform Distribu- tions}

In general, the metric $\mu_{i}$ lies in the interval $\left[\mu_{\min }, \mu_{\max }\right)$, and has a $\mathrm{CDF} F\left(\mu_{i}\right)$. We know that $F\left(\mu_{\min }\right)=0$ and $F\left(\mu_{\max }\right)=1$. When the metric is not uniformly distributed in the interval $[0,1)$, the VP-MAS and VP-MAS-PS algorithms can be easily generalized as follows.

The algorithms continue to use the state variables $\mu_{\text {base }}(k)$, $\mu_{\min }(k)$ and $\mu_{\max }(k)$ as in Sec. IV, except that they are now interpreted as percentile values. Hence, the power-mapping gets modified to $\pi\left(\mu_{i}\right)=q_{a, i}$, if $m_{i} \leq F\left(\mu_{i}\right)<m_{i+1}$, where $q_{a, i}$ is given by (16).

\section{REFERENCES}

[1] D. Tse, "Multiuser diversity in wireless networks" [Online]. Available: http://www.eecs.berkeley.edu/dtse/ima810.pdf, Apr. 2001.

[2] R. Knopp and P. A. Humblet, "Information capacity and power control in single-cell multiusercommunications," in Proc. IEEE Int. Conf. Commun., pp. 331-335, June 1995.

[3] R. Yim, N. B. Mehta, A. F. Molisch, and J. Zhang, "Progressive accumulative routing: fundamental concepts and protocol," IEEE Trans. Wireless Commun., vol. 7, pp. 4142-4154, Nov. 2008.

[4] A. Bletsas, A. Khisti, D. P. Reed, and A. Lippman, "A simple cooperative diversity method based on network path selection," IEEE J. Select. Areas Commun., vol. 24, pp. 659-672, Mar. 2006.

[5] N. Abramson, "The ALOHA system-another alternative for computer communications," in Proc. Fall Joint Computer Conf., AFIPS, p. 37, 1970.

[6] L. Kleinrock and F. A. Tobagi, "Packet switching in radio channelspart I: carrier sense multipe-acess modes and their throughput-delay characteristics," IEEE Trans. Commun., vol. 23, pp. 1400-1416, 1975.

[7] R. G. Gallager, "Conflict resolution in random access broadcast networks," in Proc. Air Force Office Scientific Research Workshop Commun. Th. Applications, pp. 74-76, Sept. 1978.

[8] B. S. Tsybakov and V. A. Mikhailov, "Random multiple packet access: part-and-try algorithm," Problemy Peredachi Infomatsii, vol. 16, pp. 6579, Oct.-Dec. 1980.

[9] M. Paterakis and P. Papantoni-Kazakos, "A simple window randomaccess algorithm with advantageous properties," IEEE Trans. Inform. Theory, vol. 35, pp. 1124-1130, Sept. 1989.

[10] A. F. Molisch, Wireless Communications. Wiley-IEEE Press, 2005.

[11] S. Verdu, Multiuser Detection. New York: Cambridge University Press, 1998.
[12] J. H. Winters, "Optimum combining in digital mobile radio with cochannel interference," IEEE J. Select. Areas Commun., vol. SAC-2, pp. 528-539, July 1984.

[13] B. Ramamurthi, A. A. M. Saleh, and D. J. Goodman, "Perfect-capture ALOHA for local radio communications," IEEE J. Select. Areas Commun., vol. 5, pp. 806-814, June 1997.

[14] J. J. Metzner, "On improving utilization in ALOHA networks," IEEE Trans. Commun., vol. COM-24, pp. 447-448, Apr. 1976.

[15] M. Sidi and I. Cidon, "Splitting protocols in presence of capture," IEEE Trans. Inform. Theory, vol. IT-31, pp. 295-301, Mar. 1985.

[16] C. Ware, Y. Wysocki, and J. F. Chicharo, "Simulation of capture behaviour in IEEE 802.11 radio modems," in Proc. VTC Fall, pp. 1393$1397,2001$.

[17] C. de Morais Cordeiro, D. Sadok, and D. P. Agrawal, "Modeling and evalution of Bluetooth MAC protocol," in Proc. Tenth Intl. Conf. Comp. Commun. Networks, pp. 518-522, 2001.

[18] M. Zorzi and Borgonovo, "Performance of capture-division packet access with slow shadowing and power control," IEEE Trans. Veh. Technol., vol. 46, pp. 687-696, Aug. 1997.

[19] X. Qin and R. Berry, "Opportunistic splitting algorithms for wireless networks," in Proc. INFOCOMM, pp. 1662-1672, 2004.

[20] Q. Zhao and L. Tong, "Opportunistic carrier sensing for energy-efficient information retrieval in sensor networks," EURASIP J. Wireless Commun. Networking, vol. 2, pp. 231-241, 2005.

[21] S. Chez, S. Verdu, and S. C. Schwartz, "Optimal decentralized control in random access multiple packet channel," IEEE Trans. Automatic Contr. vol. 34, pp. 1153-1163, Nov. 1989.

[22] V. Naware, G. Mergen, and L. Tong, "Stability and delay of finite-user slotted aloha with multipacket reception," IEEE Trans. Inform. Theory, vol. 51, pp. 2636-2656, July 2005.

[23] Q. Zhao and L. Tong, "A multiqueue service room MAC protocol for wireless networks with multipacket reception," IEEE Trans. Networking, vol. 11, pp. 125-137, Feb. 2003.

[24] Q. Zhao and L. Tong, "A dynamic queue protocol for multiaccess wireless networks with multipacket reception," IEEE Trans. Wireless Commun., vol. 3, pp. 2221-2231, Nov. 2004.

[25] W. Luo and A. Ephremides, "Power levels and packet lengths in random multiple access," IEEE Trans. Inform. Theory, vol. 48, pp. 46-58, Jan. 2002.

[26] J. Luo and A. Ephremides, "Power levels and packet lengths in random multiple access with multiple-packet reception capability," IEEE Trans. Inform. Theory, vol. 52, pp. 414-420, Feb. 2006.

[27] S. Adireddy and L. Tong, "Exploiting decentralized channel state information for random access," IEEE Trans. Inform. Theory, vol. 51, pp. 537-561, Feb. 2005.

[28] G. Ganesan and Y. Li, "Channel aware aloha with imperfect CSI," in Proc. Globecom, 2006.

[29] S. Cui, A. J. Goldsmith, and A. Bahai, "Energy-constrained modulation optimization," IEEE Trans. Wireless Commun., vol. 4, pp. 2349-2360, 2005.

[30] "Technical specification group GSM/EDGE, radio access network; radio transmission and reception," Tech. Rep. 45.005 (v7.9.0), 3rd Generation Partnership Project (3GPP), 2007.

[31] "Technical specification group radio access network; user equipment (UE) radio transmission and reception (FDD)," Tech. Rep. 25.101 (v7.7.0), 3rd Generation Partnership Project (3GPP), 2007.

[32] A. Jalali, R. Padovani, and R. Pankaj, "Data throughput of CDMAHDR: a high efficiency-high data rate personal communication wireless system," in Proc. VTC (Spring), pp. 1854-1858, May 2000.

[33] H. A. David and H. N. Nagaraja, Order Statistics. Wiley-Interscience, 3rd ed., 2003.

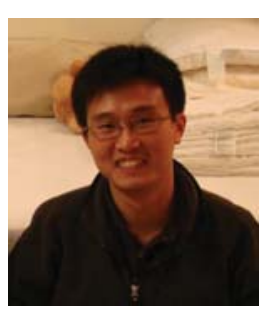

Raymond Yim (S'00-M'07) received his B.Eng. and M.Eng degrees in electrical and computer engineering from McGill University in Canada, and Ph.D. from Harvard University. In 2006-2007, he was an assistant professor in electrical and computer engineering at F. W. Olin College of Engineering, Needham, MA. Since 2008, he has been a research scientist at the Mitsubishi Electric Research Laboratories, Cambridge, MA, and has been involved in WiMAX development. His research interests include the design and analysis of cross-layered architectures and protocols for wireless communication networks, multiple access protocols, link adaptation, cooperative communications, smart antenna systems, interference management and intelligent transportation systems. 


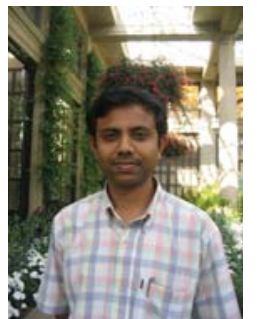

Neelesh B. Mehta (S'98-M'01-SM'06) received his Bachelor of Technology degree in Electronics and Communications Engineering from the Indian Institute of Technology, Madras in 1996, and his M.S. and Ph.D. degrees in Electrical Engineering from the California Institute of Technology, Pasadena, CA in 1997 and 2001, respectively. He was a visiting graduate student researcher at Stanford University in 1999 and 2000. He is now an Assistant Professor at the Dept. of Electrical Communication Engineering, Indian Institute of Science (IISc), Bangalore, India. Until 2002, he was a research scientist in the Wireless Systems Research group in AT\&T Laboratories, Middletown, NJ. In 2002-2003, he was a Staff Scientist at Broadcom Corp., Matawan, NJ, and was involved in GPRS/EDGE cellular handset development. From 2003-2007, he was a Principal Member of Technical Staff at the Mitsubishi Electric Research Laboratories, Cambridge, MA, USA.

His research includes work on link adaptation, multiple access protocols, WCDMA downlinks, system-level performance analysis of cellular systems, MIMO and antenna selection, and cooperative communications. He is also actively involved in radio access network physical layer (RAN1) standardization activities in 3GPP. He is a TPC co-chair for the Transmission technologies track of VTC 2009 (Fall) and the Frontiers of Networking and Communications symposium of Chinacom 2008. He is a TPC member of several conferences such as Globecom, WCNC, WISARD, and VTC. $\mathrm{He}$ is on the editorial board of the IEEE TRANSACTIONS ON WIRELESS Communications. He is also an executive committee member of the Bangalore chapter of the IEEE Signal Processing Society.

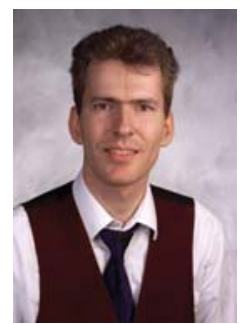

Andreas F. Molisch (S'89, M'95, SM'00, F'05) received the Dipl. Ing., Dr. techn., and habilitation degrees from the Technical University Vienna (Austria) in 1990, 1994, and 1999, respectively. From 1991 to 2000 , he was with the TU Vienna, becoming an associate professor there in 1999. From 20002002, he was with the Wireless Systems Research Department at AT\&T (Bell) Laboratories Research in Middletown, NJ. From 2002-2008, he was with Mitsubishi Electric Research Labs, Cambridge, MA, USA, most recently as Distinguished Member of Technical Staff and Chief Wireless Standards Architect. Concurrently he was also Professor and Chairholder for radio systems at Lund University, Sweden. Since 2009, he is Professor of Electrical Engineering at the University of Southern California, Los Angeles, CA, USA.

Dr. Molisch has done research in the areas of SAW filters, radiative transfer in atomic vapors, atomic line filters, smart antennas, and wideband systems. His current research interests are measurement and modeling of mobile radio channels, UWB, cooperative communications, and MIMO systems. Dr. Molisch has authored, co-authored or edited four books (among them the textbook Wireless Communications, Wiley-IEEE Press), eleven book chapters, more than 110 journal papers, and numerous conference contributions, as well as more than 70 patents.

Dr. Molisch is an editor of the IEEE Trans. Wireless Comm. and co-editor of special issues of several journals. He has been member of numerous TPCs, vice chair of the TPC of VTC 2005 spring, general chair of ICUWB 2006, TPC co-chair of the wireless symposium of Globecomm 2007, TPC chair of Chinacom2007, and general chair of Chinacom 2008. He has participated in the European research initiatives "COST 231", "COST 259", and "COST273", where he was chairman of the MIMO channel working group, he was chairman of the IEEE 802.15.4a channel model standardization group. From 2005-2008, he was also chairman of Commission C (signals and systems) of URSI (International Union of Radio Scientists), and since 2009, he is the Chair of the Radio Communications Committee of the IEEE Communications Society. Dr. Molisch is a Fellow of the IEEE, a Fellow of the IET, an IEEE Distinguished Lecturer, and recipient of several awards. 